\title{
New 4D imaging approaches with atypical sparse seismic acquisitions for reservoir monitoring
}

\section{Didier Lecerf, PGS}

Copyright 2019, SBGf - Sociedade Brasileira de Geofísica

This paper was prepared for presentation during the $16^{\text {th }}$ International Congress of the Brazilian Geophysical Society held in Rio de Janeiro, Brazil, 19-22 August 2019.

Contents of this paper were reviewed by the Technical Committee of the $16^{\text {th }}$ International Congress of the Brazilian Geophysical Society and do not necessarily represent any position of the SBGf, its officers or members. Electronic reproduction or storage of any part of this paper for commercial purposes without the written consent of the Brazilian Geophysical Society is prohibited.

\begin{abstract}
Reservoir monitoring studies need particular attention when it comes to the removal of undesirable artefacts caused by variations in seismic data acquisition. By attempting to repeat the source and receiver geometries between surveys, as precisely as possible, any subsequent $4 \mathrm{D}$ noise is minimized.
\end{abstract}

However, in some cases it is uneconomic or not possible to repeat the survey geometries between vintages. This is the case when a different OBN seabed layout is used or a towed streamer survey is combined with an OBS acquisition.

We are presenting an image domain approach that builds on wave equation reflectivity inversion using Point Spread Functions (PSFs) for correcting illumination differences between 4D datasets. In this two-step least-squares imaging method, the reflectivity is recovered by explicitly computing multi-dimensional PSFs using wave-equation modelling and de-convolving these PSFs with the final migrated image. We define a 4D formulation, which is not dependent on geological and/or reservoir production constraints by introducing the concept of cross-survey PSFs (XPSFs).

The presented 4D imaging concept is suitable for any time-lapse acquisition geometries (repeated or not) but shows its full potential for sparse non-repeated acquisition.

\section{Introduction}

By repeating the source and receiver geometries, and acquisition parameters between time-lapse surveys, as precisely as possible, any subsequent 4D noise is minimized. However, in some cases it is uneconomic or not possible to repeat the survey geometries between vintages. This is the case when a different OBN layout is used or when various streamer acquisition azimuths are involved or when a towed streamer survey is compared with an OBS acquisition.

Some solutions to this problem have been described in the literature for implementations in both the data and image domain. For reconciling steamer and OBS data, intelligent $4 \mathrm{D}$ binning in the data domain can be performed by selecting trace pairs based on multiple criteria such as the Common Depth Point (CDP) distance and/or pseudo-incidence angle and azimuth similarities at the reservoir level. Dip-angle image filtering has also been suggested in order to better reconcile towed streamer and OBS migrated images by Haacke et al. (2017).

The image domain approach for correcting illumination differences between 4D datasets, that we present here, builds on previously published work concerning wave equation reflectivity inversion using Point Spread Functions (PSFs). In this two-step least-squares imaging method, the reflectivity of depth migrated images is recovered by explicitly computing multi-dimensional PSFs using wave-equation modeling and de-convolving these PSFs with the final migrated image (Valenciano et al., 2006). Ayeni and Biondi (2010) have previously exploited the PSF concept for 4D reservoir monitoring using a target-oriented joint least-squares migration approach with spatial and temporal constraints.

The aim of the study presented here is to define a 4D formulation which is not dependent on geological and/or reservoir production constraints. We modified the previous joint inversion formulations by introducing the concept of cross-survey PSFs (XPSFs). The benefit of working in the image domain is that the illumination variation can be directly evaluated and compensated for at any location in the 4D image regardless the acquisition geometries.

\section{D reflectivity inversion using XPSFs}

As shown by Valenciano et al. (2006), a least-squares inversion framework can be used for inverting the reflectivity in the image domain. The recorded seismic data $\mathbf{d}$ can be expressed as a linear modeling operator $\mathbf{L}$ working on the subsurface reflectivity $r: d=L r$

The reflectivity $\mathbf{r}$ therefore can be estimated using the Hessian matrix $\mathbf{H}=\mathbf{L}$ ' $\mathbf{L}$, where $\mathbf{L}^{\prime}$ is the migration operator (or adjoint to the modeling operator).

$$
\hat{r}=\left(L^{\prime} L\right)^{-1} L^{\prime} d_{o b s}=H^{-1} m_{m}
$$

$$
\begin{aligned}
& \text { Where } \\
& m_{m}=\text { migrated image, } \\
& \hat{r}=\text { estimated reflectivity, } \\
& d_{\text {obs }}=\text { observed data }
\end{aligned}
$$

The main difficulty of solving the above equation is the need to explicitly compute the inverse of the Hessian matrix. Using PSFs, the Hessian $\mathrm{H}$ can very efficiently be 
computed in a target-oriented fashion as a set of local imaging responses of a scatter point grid.

\section{D joint reflectivity inversion summary}

In a 4D time-lapse experiment, a matrix system can be defined using $\hat{r}_{0}, \hat{r}_{1}$ and $\widetilde{m}_{0}, \widetilde{m}_{1}$ respectively estimated reflectivity models and migrated images for the base and monitor surveys. By introducing the cross-survey Hessian term $\mathrm{HiH}_{\mathrm{i}}$, the 4D joint inversion system (with no temporal/geological constraints) can be described as follow:

$$
\left(\left[\begin{array}{cc}
H_{1} H_{0} & 0 \\
0 & H_{0} H_{1}
\end{array}\right]+\left[\begin{array}{cc}
\varepsilon_{01} & 0 \\
0 & \varepsilon_{10}
\end{array}\right]\right)\left[\begin{array}{l}
\hat{r}_{0} \\
\hat{r}_{1}
\end{array}\right]=\left[\begin{array}{l}
H_{1} \tilde{m}_{0} \\
H_{0} \tilde{m}_{1}
\end{array}\right]
$$

With the regularization terms:

$$
\varepsilon_{01}=\lambda_{01}\left\langle\left(\mathbf{H}_{0} \mathbf{H}_{1}\right)^{*} \cdot \mathbf{H}_{0} \mathbf{H}_{1}\right\rangle \text { and } \varepsilon_{10}=\lambda_{10}\left\langle\left(\mathbf{H}_{\mathbf{1}} \mathbf{H}_{0}\right)^{*} \cdot \mathbf{H}_{1} \mathbf{H}_{0}\right\rangle
$$

The $4 \mathrm{D}$ reflectivity change is given by: $\Delta \hat{r}=\hat{r}_{1}-\hat{r}_{0}$

The diagonal of the cross-survey Hessian corresponds to the cross-survey illumination. If one of the surveys does not illuminate a particular reflector sufficiently than the cross-survey illumination becomes very small. In contrast, if the wavefields of the base and monitor are similar at the reflector then the cross-survey illumination is maximum. The cross-survey Hessian will be represented by a set of XPSFs, which are derived from the PSFs. As a result, the illumination common to both datasets is honored to compute the changes in reflectivity caused by production effects.

\section{D synthetic example 1: Sigsbee2B model}

The first 4D synthetic data example has been created using a subset of the Sigsbee2B 2D model. The 4D synthetic seismic data have been generated by modeling two different types of acquisitions, a streamer acquisition for the base survey and an OBN acquisition for the monitor survey (Figure 1).

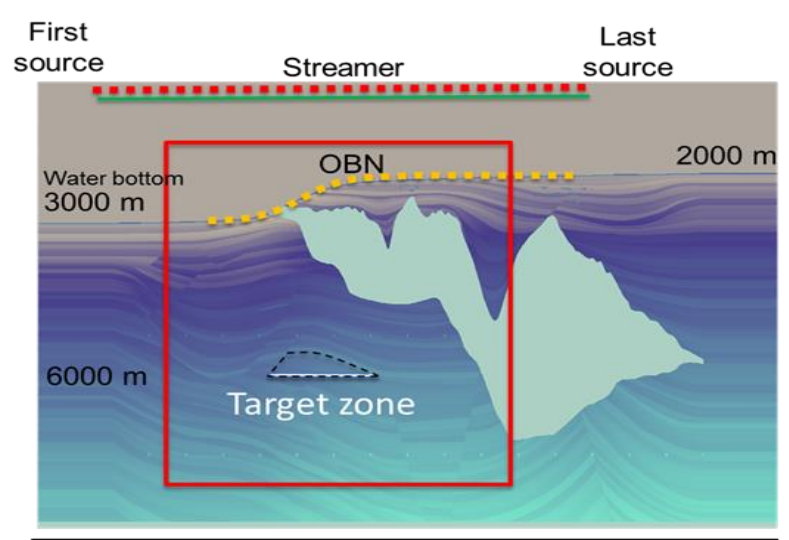

Figure 1: Sigbee2B model for Towed Streamer Base and OBN Monitor surveys

The baseline towed streamer acquisition is modelled using 500 shots with a shot point distance of $50 \mathrm{~m}$ and a $6,000 \mathrm{~m}$ long sensor cable with a receiver distance of 20 $\mathrm{m}$. The monitor dataset has been modelled using the same shots geometry as for the base survey (i.e. a total of 500 shots) but the receiver network was composed of 120 OBNs positioned on the seabed $200 \mathrm{~m}$ apart from each other. The modelled 4D changes at the reservoir are characterized by a $40 \mathrm{~m}$ shift of the position of the Oil Water Contact (OWC) and a velocity increase at the reservoir location of $10 \%$. It should be noted that no extra $4 \mathrm{D}$ processing steps were applied to the data pre or postimaging.

The high values in the cross-survey illumination panel shown in Figure 2c define area where the illuminations of the two acquisitions has been very similar. The reservoir has been purposely located beneath the salt body in an area of significant salt thickness variation. The aim is to evaluate the ability of the proposed cross-survey joint reflectivity inversion to recover the true $4 \mathrm{D}$ reflectivity without introducing unwanted artefacts, which can be interpreted as 4D noise.

a) Illumination Streamer

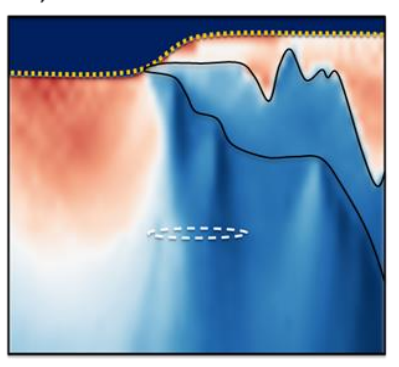

c) Cross-Survey Illumination

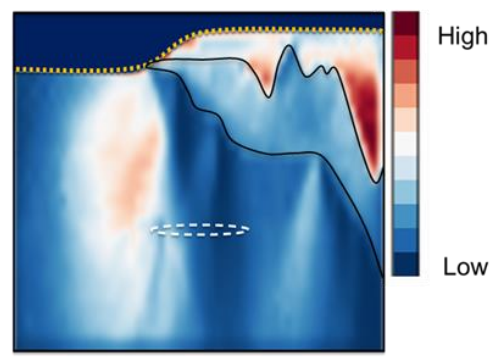

Figure 2: Illumination a) Streamer, b) $O B N$, c) Cross-Survey

The results of the reflectivity inversion are presented in Figure 3 for two different inversion approaches. In the first approach, a least-squares reflectivity inversion is carried out separately for both datasets. In the second approach, the new joint reflectivity inversion process is applied.

Despite the very different acquisition geometries of both base and monitor surveys, the inversion process in both cases has been able to recover a meaningful 4D reflectivity response. However, the separate inversion approach has resulted in more reflectivity artefacts 
around the OWC, which are unrelated to the true 4D signal. The joint inversion approach has produced results that show less artefacts especially in the area outside the reservoir where no production has occurred.

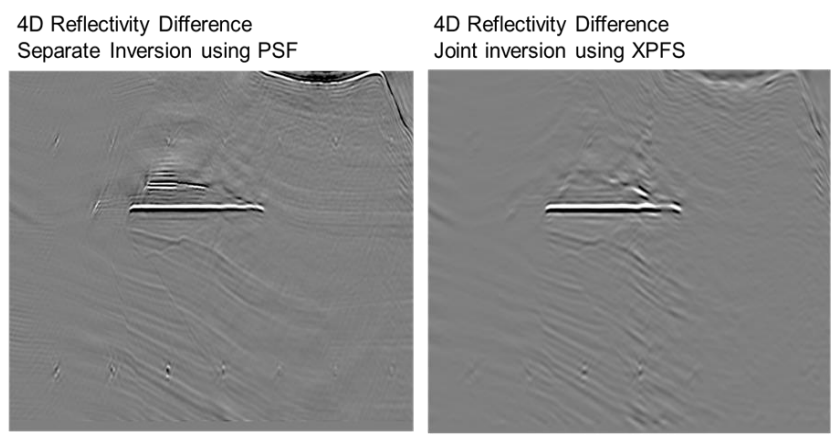

Figure 3: 4D reflectivity difference using separate inversion (left) and joint inversion (right)

\section{D synthetic example 2: SEAM time-lapse model}

The second synthetic example has been generated using the 3D/4D reservoir model provided by the SEAM timelapse project (Oristaglio, 2016; Smit et al., 2017). The 4D modelling involves two non-repeated OBN geometries. The base survey design comprised 60 OBNs laid out in a rectangular shape and the monitor survey included two squares of 30 nodes each that are located to the side of the base survey. The shot carpet is similar for both vintages. A strong 4D difference is expected at the GasOil contact at $3400 \mathrm{~m}$ depth due to the large velocity variation as indicated in the Figures $4 \mathrm{~b}$ and $4 \mathrm{c}$.

a) Design OBN surveys Non-Repeated (2x60)

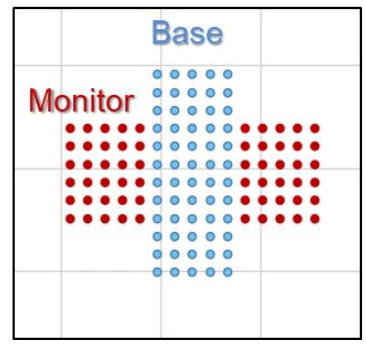

c) 4D SEAM pilot model

Depth slice

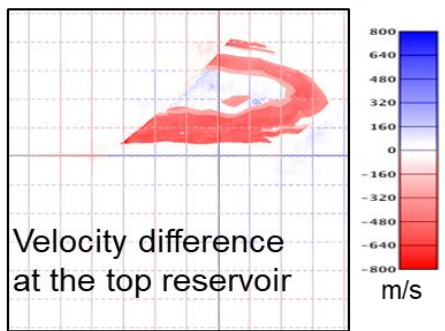

Figure 4: a) Design of non-repeated OBN geometries. b) Section of the SEAM $4 D$ pilot velocity model. c) Depth slice at the top reservoir $(3400 \mathrm{~m})$ of the $4 D$ velocity variation.
Figure 5 displays the 4D results for depth slices at the water bottom (i.e. $260 \mathrm{~m}$ ) and at the top of the reservoir (i.e. $3400 \mathrm{~m}$ ). Figure $5 \mathrm{a}$ shows the 4D seismic difference results (i.e. straight seismic amplitude difference), Figure $5 b$ show respectively the separate reflectivity inversions and the joint reflectivity inversion results. At the water bottom, it can be observed that the 4D difference around the node locations appear more focused with the reflectivity inversion applied. However, the reflectivity differences do not cancel out for the separate reflectivity inversion case, as the individual illumination corrections are unconnected. In contrast, the jointly inverted reflectivity change (i.e. 4D reflectivity differences) becomes negligibly small at the water bottom; a consequence of the use of the cross-survey PFSs.

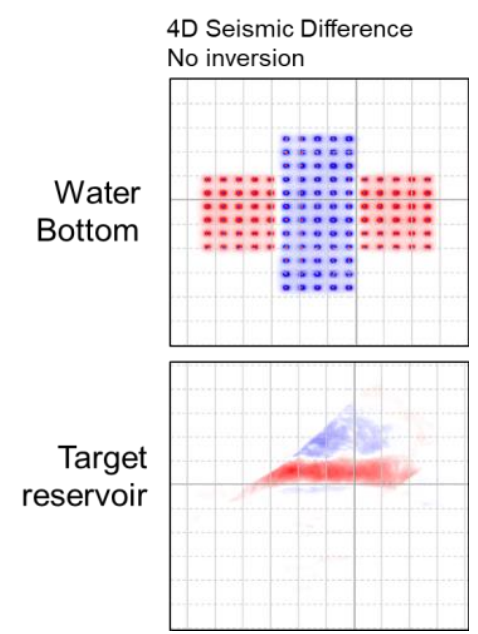

Figure 5a: Depth slices of the $4 D$ seismic difference at the water bottom (top) and at the top reservoir (bottom).

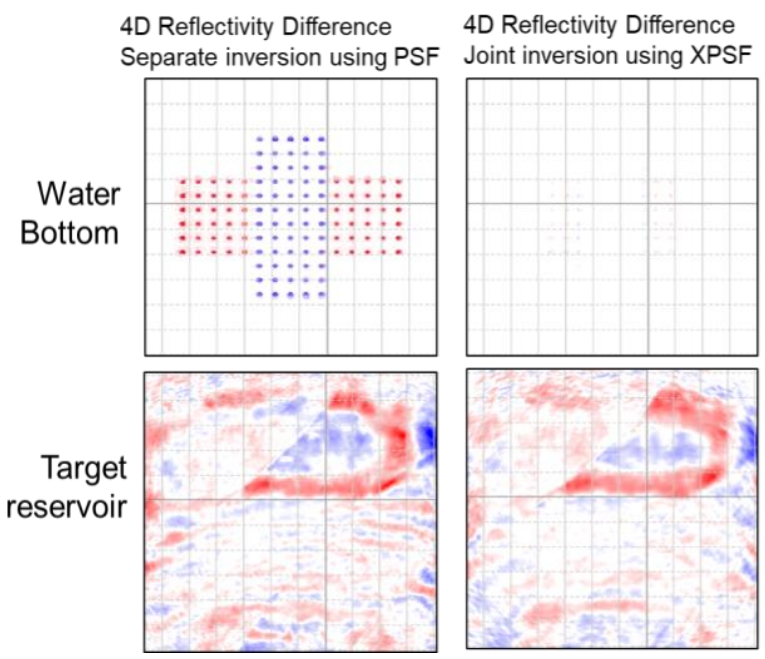

Figure 5b: Depth slices of the $4 D$ reflectivity difference at the water bottom (top) and at the top reservoir (bottom). 
The 4D signal at the reservoir level (Figure 5a) is partially imaged by the seismic amplitude difference and presents large lateral amplitude variations. The separate reflectivity inversions have improved the 4D signal but the lack of a 4D constraint has over-boosted the 4D noise outside and inside of the ' $D$ shaped' reservoir area. The joint reflectivity inversion was able to reduce the illumination induced 4D noise further and enable the recovery of a consistent 4D signal amplitude with the same geometry as the original 4D velocity model (Figure 4c).

\section{Conclusion}

Image domain 4D reflectivity inversion using multidimensional PSFs has been shown to compensate for significant illumination differences when different survey geometries are used for 4D imaging. The proposed methodology is advantageous in 4D studies where the geometry of the different acquisitions cannot be replicated. This is the case when towed streamer surveys with different acquisition directions are used in a 4D experiment or when streamer and OBN (Ocean Bottom Node) surveys are combined.

As shown using synthetic data examples, the joint reflectivity inversion process delivers superior results when compared to separate inversions as it ensures a more robust recovery of the 4D effects. The presented new methodology using cross-survey Point Spread Functions (XPSFs) ensures consistency of the wavefields for recovering the $4 \mathrm{D}$ signal due to the reservoir production.

The 4D synthetic data examples shown here use models that produce strong and easy to detect $4 \mathrm{D}$ signals. If more subtle 4D effects need to be recovered, it will be paramount to repeat the acquisition geometries between the different time-lapse surveys as closely as possible.

The joint inversion methodology using XPSFs described here, although still beneficial when applied to repeat surveys of largely similar geometry. Combining this 4D imaging inversion technique with an optimum 4D repeated acquisition design will be preferred when subtle 4D effects are to be detected.

\section{Acknowledgements}

The author would like to acknowledge Elena Klochikhina, Alejandro Valenciano and Nizar Chemingui (PGS) for their support and constructive discussions during this project and PGS management for their approval to publish this work. In addition, special thanks goes to Martin Besselievre which has substantially contributed to this work.

\section{References}

Ayeni, G., and Biondi, B. [2010] Target-oriented joint least-squares migration/inversion of time-lapse seismic data sets. 2010, Geophysics, 75(3) R61-R73.
Haacke, R. R., Casasanta, L., Hou, S. and Henderson J. R. [2017] Dip-angle image filtering for 4D processing of towed-streamer and OBN datasets, First EAGE Workshop on Practical Reservoir Monitoring, Th PRM 07.

Oristaglio, M.L., SEAM Update: Integrated reservoir and geophysical modelling: SEAM Time Lapse and SEAM Life of Field, [2016] The Leading Edge, 35(10), 912-915.

Smit, D., Oppert, S., Stefani, J., Artus, V., Herwanger, J., Popov, P., Bottrill, A., Tan, L., Hu, W., Liu, J., Abriel, W., Detomo, R., Barkhouse, W., and Oristaglio, M.L. [2017] Large scale numerical simulation of reservoir monitoring SEAM Time Lapse, First EAGE Workshop on Practical Reservoir Monitoring,, Th PRM 13.

Valenciano, A.A., Biondi, B., and Guitton, A., [2006] Target oriented wave-equation inversion, Geophysics, 71(4) A35-A38. 